\title{
OCORRÊNCIA DE CHAMOSITA NOS SEDIMENTOS RECENTES DA BAIA DE ARATU (BA)
}

\author{
ABÍLIO CARLOS DA SILVA PINTO BITTENGOURT* e \\ GERALDO DA SILVA VILAS BOAS*
}

\begin{abstract}
Authigenic chamosite, comprising pellets and internal molds of algae Halimeda, gasteropods, foraminifers, etc., has been identified by X-ray diffraction and chemical analyses of recent sediments from the "Baía de Aratu".

Sedimentation of clay prevails in the restricted, low energy environment. Chamosite occurs in depths ranging from 5 to $30 \mathrm{~m}$. This is the first report on chamosite at the Brazilian coast.

Associated iron minerals are represented by goethite and siderite. These sediments with chamosite may represent the last transgressive phase of the Quaternary, characterized by slow and fine sedimentation.
\end{abstract}

INTRODUÇÃo Em recentes estudos realizados na Baía de Aratu (Fig. 1), foi constatada a presença de chamosita autigênica em algumas amostras coletadas no fundo, sendo esta a primeira ocorrência registrada no Brasil (Fig. 2).

A chamosita é um filosilicato com estrutura do tipo caulinita-serpentina. É um mineral essencialmente trioctaédrico com a seguinte fórmula aproximada: $\left(\mathrm{Fe}^{2+}, \mathrm{Mg}\right)_{2 \cdot 3}$ $\left(\mathrm{Fe}^{3+}, \mathrm{Al}\right)_{0.7}\left(\mathrm{Si}_{1.4} \mathrm{Al}_{0.6}\right) \mathrm{O}_{5}(\mathrm{OH})_{4}$ (Brindley, 1961). Em muitos minérios de ferro sedimentares, a chamosita constitui o principal mineral de argila presente. A maioria dos autores concorda que a chamosita se forma contemporaneamente à sedimentação ou logo nos primeiros estágios diagenéticos, em ambiente marinho raso.

Até os estudos feitos por Porrenga (1967), as ocorrências conhecidas de chamosita recente estavam restritas aos ambientes marinhos rasos, nos trópicos, com lâminas de água inferiores a $60 \mathrm{~m}$ e com temperatura da água superior a $20^{\circ} \mathrm{G}$. Rohrlich et alli (1969) encontraram chamosita recente, na forma de pelotas fecais, em sedimentos não-consolidados do Loch Etive (Escócia), um ambiente marinho restrito com temperatura média das águas de $8{ }^{\circ} \mathrm{C}$. A Fig. 2 mostra um mapa (adaptado de Porrenga, 1967) indicando as ocorrências de chamosita recente nos sedimentos marinhos atuais.

OCORRENCIA DA CHAMOSITA A Baía de Aratu (Fig. 1) é uma pequena entrada de mar (cerca de $20 \mathrm{~km}^{2}$ ), situada no bordo do lado leste da Baía de Todos os Santos e ligada à mesma por um canal estreito e profundo. Em seu corpo principal, as profundidades são inferiores a $10 \mathrm{~m}$, enquanto que, no canal, podem atingir até $50 \mathrm{~m}$. Em seus bordos, recortados, encontram-se três pequenos rios e uma série de riachos que contribuem para a sedimentação, em sua maioria com material fino originado da alteração das rochas lutíticas cretácicas, aflorantes na região.

A Baía de Aratu pode ser considerada como um ambiente de águas calmas, com fraca energia. Os sedimentos do fundo são predominantemente argilosos, com pouco silte e porcentagens variadas da fração grosseira (>62 mícrons) representada por fragmentos de diversos organismos: moluscos, equinodermas, alga Halimeda, cirrípedos, foraminíferos e pequenas conchas inteiras de gasterópodos e lamelibrânquios. Os teores de

* Programa de Pesquisa e Pós-Graduação em Geofísica e Instituto de Geociências da UFBa, Rua Caetanó Moura, 123 - Federação - 40 000, Salvador (BA) 


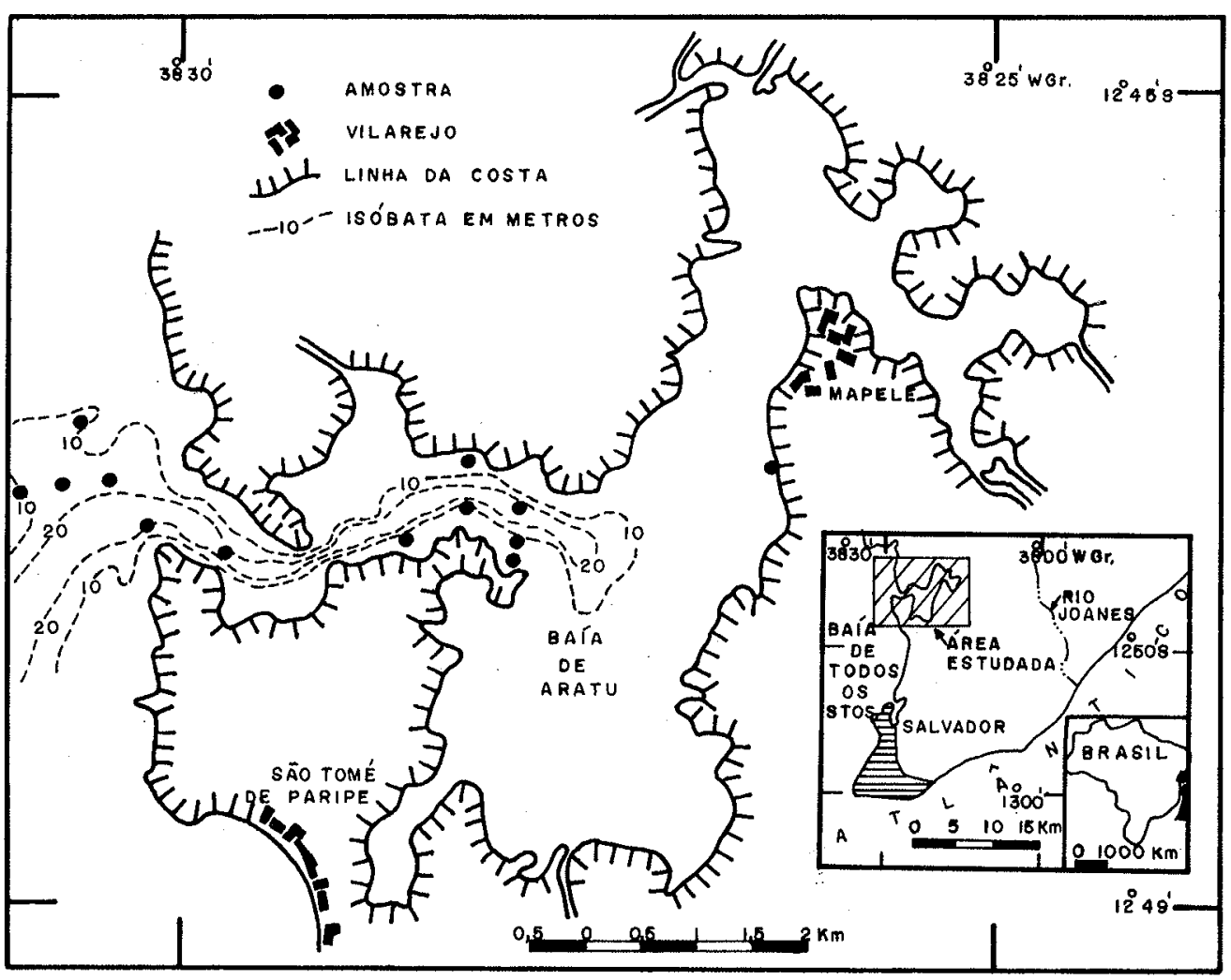

Figura 1 - Mapa de localização das amostras com chamosita na Baía de Aratu

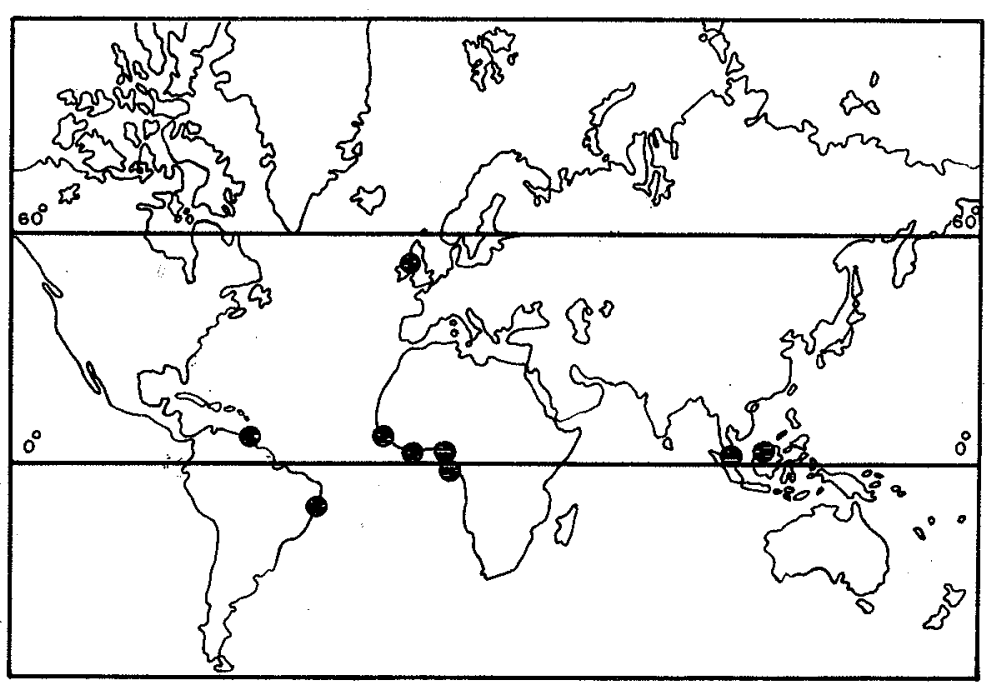

Figura 2 - Mapa de localização das ocorrências de chamosita em sedimentos marinhos atuais (adaptado de Porrenga, 1967) 
matéria orgânica nos sedimentos apresentam, em média, um valor de 2,5\%. A temperatura média anual da água é superior a $20^{\circ} \mathrm{C}$ e a salinidade, embora se disponha somente de indicações gerais, é da ordem de 34 a $35 \%$.

A Fig. 1 apresenta a localização das amostras em que foi verificada a presença de chamosita na Baía de Aratu. As amostras estão concentradas na zona do canal e em sua frente, em profundidades variando de 5 a $30 \mathrm{~m}$; no corpo principal da baía, unicamente uma amostra apresentou a chamosita, a uma profundidade de $3 \mathrm{~m}$.

A chamosita aparece nas frações de 500-250 (na maioria dos casos, como preenchimento de gasterópodos), 250-125 e 125-62 mícrons, com porcentagens em peso, em relação ao total da fração grosseira, variando entre 0,01 a 6,0\%. Apenas uma amostra, coletada na frente da entrada da baía, apresentou uma porcentagem elevada, de 17,40\%, em peso.

Os grãos aparecem com duas colorações bem distintas: (a) verde-escuros, quase-pretos, apresentando lobos separados por sulcos e ranhuras, e (b) verde-claros, acinzentados, com aspecto superficial uniforme (liso). A chamosita se apresenta de três maneiras bem-marcadas, bem-semelhantes aos modos de ocorrência da glauconita: como preenchimento de organismos diversos, em formas pelotoidais ou, ainda, disformes (free-form). Na tentativa de melhor expressar as diferentes formas de ocorrência, foi feito um estudo estatístico, segundo os diferentes tipos morfológicos, adotando-se como base a classificação utilizada por Burst (1958) para grãos de glauconita. Os resultados estão representados na Tab. I.

Por essa tabela, vê-se que a grande maioria dos grãos observados apresenta aspecto disforme. Entre os grãos alongados e semi-esféricos (Fig. 3), alguns apresentam suturas

Tabela I - Formas de ocorrências dos grãos de chamosita na Baía de Aratu

\begin{tabular}{|lc|}
\hline FORMA & PORCENTAGEM \\
\hline \hline Alongados & 12,20 \\
Semi-esféricos & 19,00 \\
Disformes ("free-form") & 56,80 \\
Preenchimento de organismos & 12,00 \\
(formas variadas) & \\
\hline
\end{tabular}

Figura 3 - Grãos alongados e semi-esféricos de chamosita: (a) pelotas de coloração verde-clara acinzentada, com aspecto superficial uniforme (liso); (b) grãos alongados de coloração verde-escura, quase-preta, com lobos separados por sulcos e ranhuras

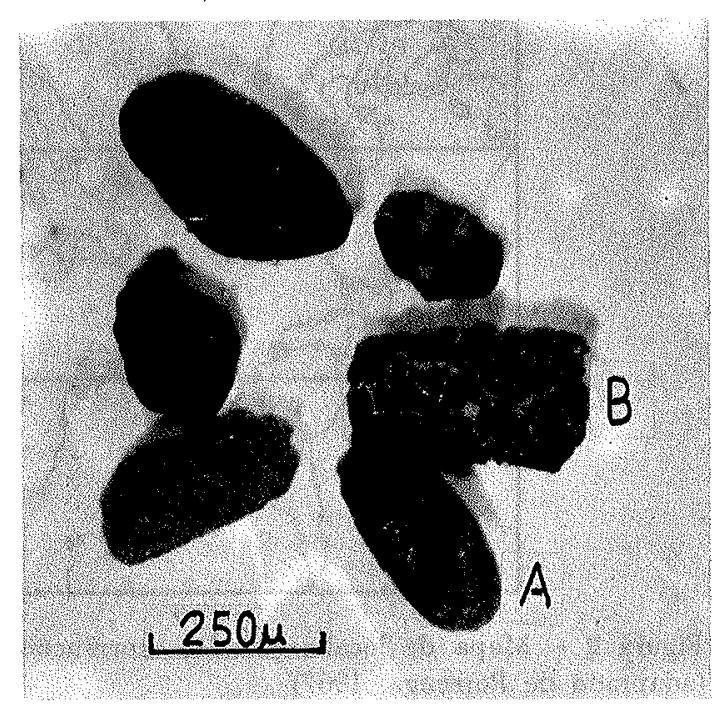


e enrolamentos, sugerindo pelotas fecais; a maioria, entretanto, não apresenta nenhuma decoração, tornando dificil, desse modo, determinar sua origem. Burst (1958), estudando grãos de glauconita, sugere, para esses grãos alongados e semi-esféricos sem decoração exterior, as seguintes origens: (a) pelotas fecais que foram depositadas sem marcas exteriores, (b) pelotas fecais que perderam suas decorações através da abrasão ou (c) a simples aglomeração de pelotas de folhelhos e de argilas do fundo, por correntes de fundo ondulatórias...". Mais provavelmente, os grãos encontrados na Baía de Aratu devem ter suas origens ligadas à primeira e à última possibilidades apresentadas por Burst (1958), tendo em vista o fato de o ambiente ser de energia muito fraca, como também pelo fato de os grãos não apresentarem evidências de abrasão. Por fim, aproximadamente $12 \%$ dos grãos apresentam formas variadas tipicamente orgânicas, resultado da conversão em moldes de materiais que preencheram cavidades de organismos. A chamosita foi encontrada preenchendo, principalmente, conchas de gasterópodos, ostracodes, carapaças de foraminíferos (Elphidium) e canais dos segmentos de alga Halimeda (Figs. 4, 5, 6, 7 e 8). O preenchimento da Halimeda é em caso excepcional, porquanto, pela literatura que se dispõe, a chamosita, do mesmo modo que a glauconita, só tem sido encontrada preenchendo carapaças de organismos animais.

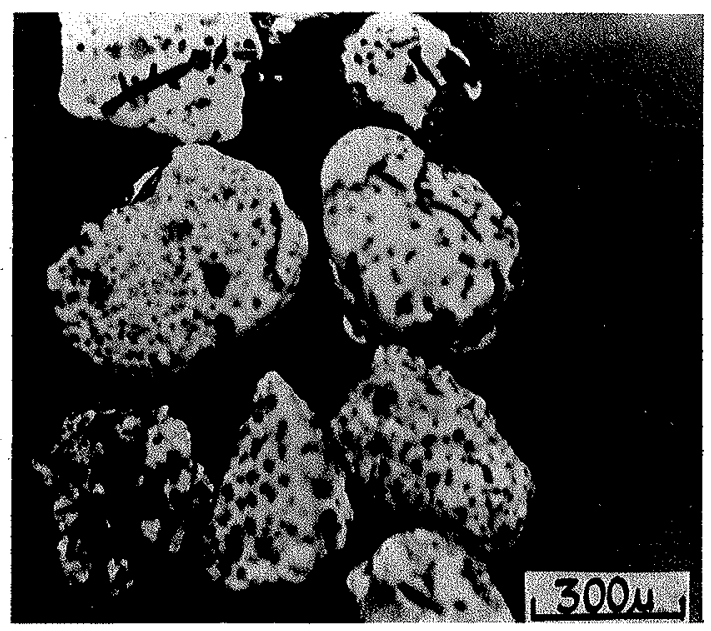

Figura 4 - Algas do tipo Halimeda, apresentando preenchimento de chamosita (parte escura)

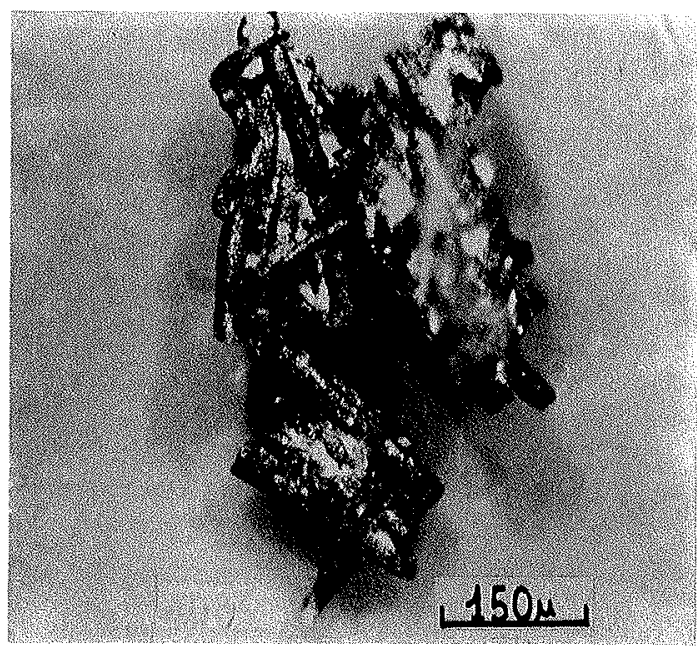

Figura 5 - Molde dos canais de segmento de alga Halimeda preenchidos por chamosita. As partes brancas indicam os restos do material da Halimeda já quase que completamente dissolvidos 
Figura 6 - Preenchimento de chamosita em valvas de ostracodes

Figura 7 - Preenchimento de chamosita em foraminíferos do gênero Elphidium. As conchas apresentam-se parcialmente dissolvidas
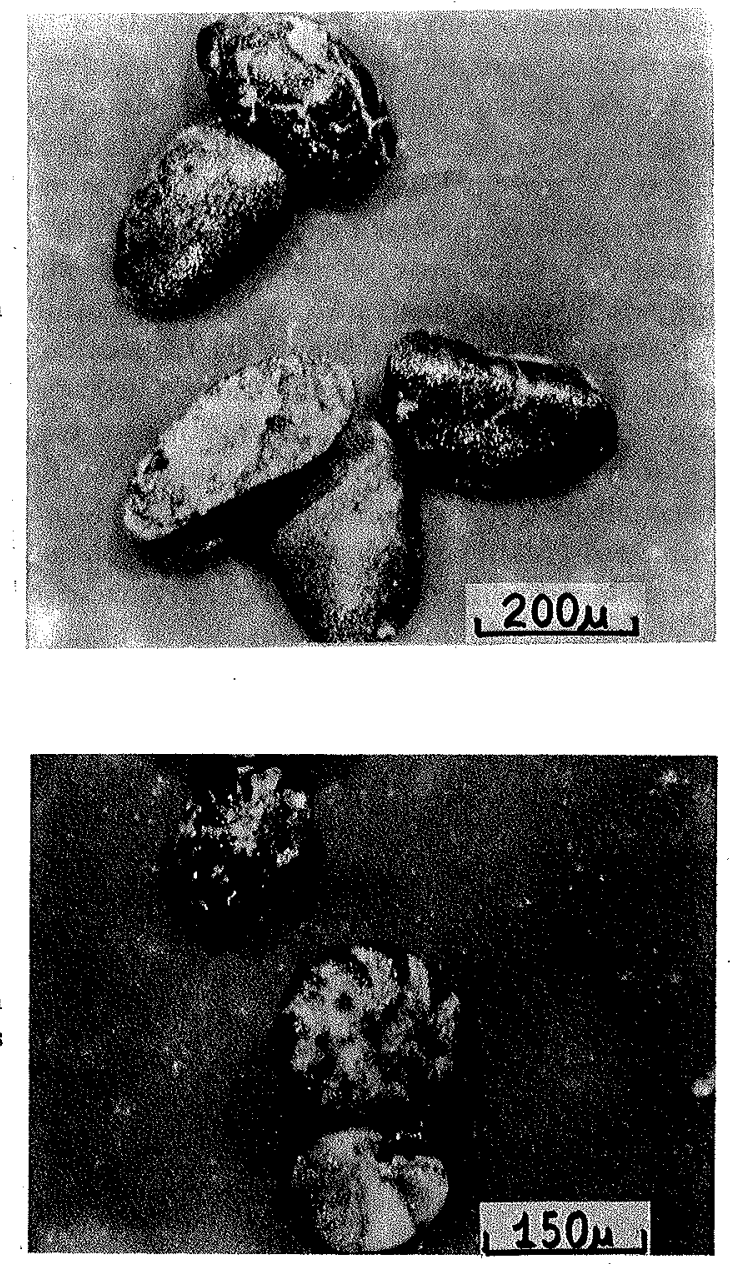

Figura 8 - Preenchimento de chamosita em gasterópodos. As conchas apresentam-se parcialmente dissolvidas

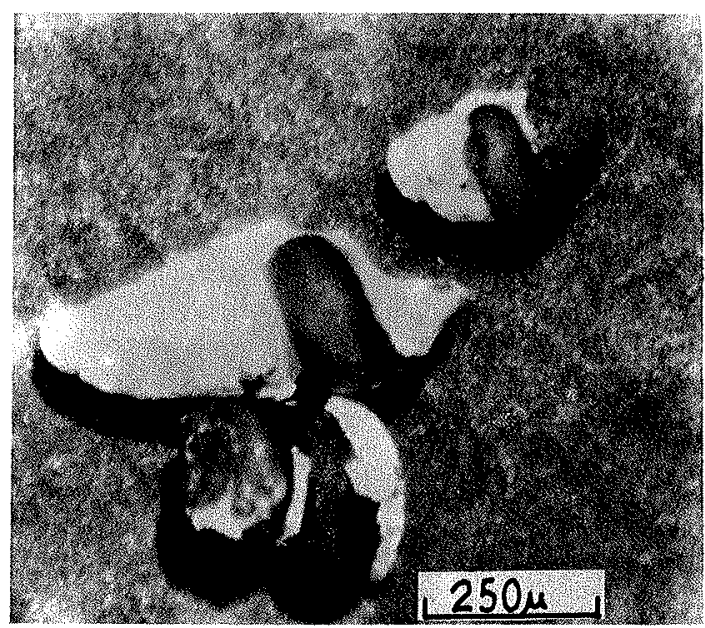


Os estudos mineralógicos na fração grosseira evidenciaram a presença, no corpo principal da Baía de Aratu, ainda que em poucas amostras e em pequenas porcertagens, de pequenos grãos arredondados de goetita e de fragmentos de conchas com incrustações de pequenos cristais de siderita. Porrenga (1965) encontrou, no delta do Niger, próximo às praias e em profundidades inferiores a $10 \mathrm{~m}$, pelotas marrons de goetita e, ocasionalmente, pequenas quantidades de chamosita. Em Aratu, a goetita aparece em quantidade apreciável somente em três amostras, sendo que, em duas delas, próximas às desembocaduras de rios e com profundidades em torno de $1 \mathrm{~m}$, não está associada à chamosita e, na terceira, com uma profundidade de $25 \mathrm{~m}$, encontra-se associada a ela. Ainda Porrenga (1967) considera que as pelotas de geothita podem ser produtos de oxidação de grãos retrabalhados de chamosita. Strakhov (1957) observa que a chamosita habitualmente, nas rochas, associa-se à siderita. Em Aratu, a chamosita e a siderita não chegam a ser encontradas em uma mesma amostra, porém, em locais próximos.

ANÁLISE PETROGRÁFICA,DIFRAÇÃO DE RAIOS X E ANÁLISE QUÍMICA Em lâmina delgada, tanto as pelotas quanto os moldes de organismos apresentam coloração verde amarelada, muito ligeiramente pleocróica. Formam aglomerados de grânulos submicrocristalinos com birrefringência próxima à do quartzo. Em alguns grãos, aparecem placas de mineral micáceo, de cor verde muito clara, apresentando, em alguns casos, cor de interferência anômala e, em outros, cor de alta birrefringência, sendo, possivelmente, minerais do tipo clorita e biotita.

Não foi observada, nas pelotas de chamosita da Baía de Aratu, evidências de estrutura concêntrica, como descritas por Rohrlich et alli (1969) em pelotas de chamosita de Loch Etive, nem anéis envoltórios de goethita, como mencionado por Porrenga (1965) para as amostras do delta do Niger.

As amostras, após o tratamento com $\mathrm{H}_{2} \mathrm{O}_{2}$ a 100 vol., foram misturadas e passadas em separador magnético para a concentração dos minerais de ferro. Através do processo de catação, sob a lupa binocular, foram separadas da fração magnética as pelotas e moldes de organismos, a fim de serem efetuados estudos por difração de raios $\mathrm{X}$. $\mathrm{O}$ padrão de difratometria dos raios X (Fig. 9) mostra reflexões atribuídas à chamosita (picos de $7,08 \AA ; 4,55 \AA ; 3,56 \AA ; 2,595 \AA ; 2,44 \AA$; e $2,13 \AA$ ), bastante semelhantes ao padrão obtido por Rohrlich et alli (1969). Analogamente ao observado por Porrenga (1965) e Martin (1972), em sedimentos recentes dos deltas do Niger e do Orinoco e na plataforma continental da Costa do Marfim, os diagramas de raios $\mathrm{X}$ da chamosita da Baía de Aratu apresentam picos bastante largos, evidenciando uma estrutura pouco ordenada. Associados a chamosita, aparecem a montmorilonita e o quartzo (Fig. 9).

A composição química de pelotas fecais de chamosita da Baía de Aratu foi determinada por absorção atômica. Os resultados são similares às análises de chamositas recentes compilados da literatura (Tab. II).

CONCLUSóES A descoberta da chamosita na Baía de Aratu representa uma contribuição ao conhecimento da distribuição desse mineral nas imediações do Equador. Na América Latina, a chamosita foi encontrada pela primeira vez, em sedimentos recentes, ao norte do Equador, no golfo de Paria, sendo a segunda ocorrência, na Baía de Aratu, a mais meridional até então conhecida (Fig. 2).

Os grãos se apresentam como preenchimento de organismos animais e vegetais ou em formas peletoidais ou, ainda, disformes (free-form). É interessante evidenciar o fato de que, na Baía de Aratu, é observada pela primeira vez o preenchimento de algas do tipo Halimeda. 


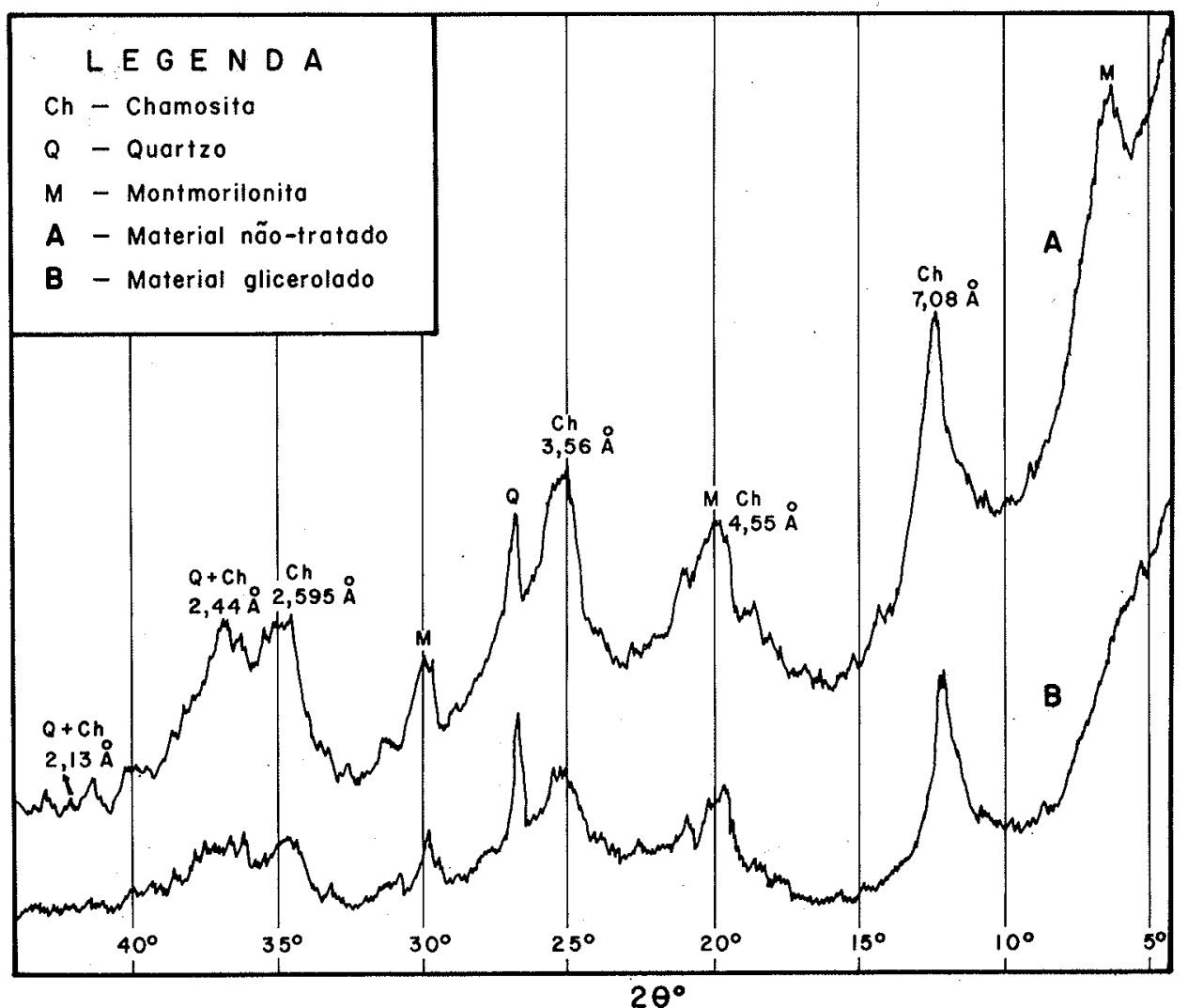

Figura 9 - Difratograma de raios-X da chamosita da Baía de Aratu

A chamosita, na Baía de Aratu, em seu aspecto externo, é muito semelhante à glauconita, só podendo ser diferenciada da mesma por intermédio da difração dos raios $\mathrm{X}$.

Com exceção da ocorrência de Loch Etive, Escócia, onde a chamosita foi encontrada em ambiente marinho restrito com águas de baixa temperatura, a chamosita tem sido considerada como originária de áreas próximas a deltas, onde a concentração de ferro nas águas é alta, provavelmente devido ao suprimento dos rios, e onde a agitação das ondas e das correntes induz acrescimentos concêntricos ao redor de um núcleo. Na Baía de Aratu, o ambiente é marinho restrito, sem entretanto apresentar características deltáicas. Os pequenos riachos que desembocam nessa baía atualmente trazem material fino. em suspensão. Devido à fraca energia na área, a formação de acrescimentos concêntricos ao redor de um núcleo não deve ser esperada.

Porrenga (1967) aponta o fato da chamosita ocorrer em profundidades inferiores a $60 \mathrm{~m}$. Na Baía de Aratu, a chamosita aparece entre profundidades de 3 a $30 \mathrm{~m}$.

Informações de poços perfurados na Baía de Aratu indicam uma espessura de sedimentos de até $30 \mathrm{~m}$, constituídos de material predominantemente argiloso, misturado com conchas. Isso dá uma idéia de que se verificou, durante o Quaternário, uma transgressão, invadindo o mar toda a baía. Nesse caso, analogamente ao que tem sido observado a partir da glauconita (Triplehorn, 1966), a chamosita, em Aratu, pode sugerir uma fase transgressiva do Quaternário, associada a uma lenta sedimentação.

A chamosita, na forma de preenchimentos, indica claramente, uma origem diage- 
Tabela II - Composição química das pelotas fecais de chamosita da Baía de Aratu e de chamositas analisadas por outros autores

\begin{tabular}{|c|c|c|c|c|c|}
\hline & 1 & 2 & 3 & 4 & 5 \\
\hline $\mathrm{SiO}_{2}$ & 31,43 & 32,00 & 52,00 & 46,00 & 32,00 \\
\hline $\mathrm{Al}_{2} \mathrm{O}_{3}$ & 9,86 & 10,05 & 8,00 & 13,00 & 15,50 \\
\hline $\mathrm{Fe}_{2} \mathrm{O}_{3^{\prime}}$ & 34,31 & 38,10 & 20,00 & 23,00 & 34,00 \\
\hline $\mathrm{FeO}$ & nd & nd & nd & nd & nd \\
\hline $\mathrm{MgO}$ & 3,70 & 4,70 & 8,30 & 4,70 & 4,00 \\
\hline $\mathrm{CaO}$ & 0,88 & 0,72 & 0,50 & 0,50 & nd \\
\hline $\mathrm{Na}_{2} \mathrm{O}$ & 0,15 & nd & 0,30 & 0,40 & 0,20 \\
\hline $\mathrm{K}_{2} \mathrm{O}$ & 2,35 & 1,30 & $<0,50$ & $<0,50$ & $<0,50$ \\
\hline $\mathrm{TiO}_{2}$ & nd & 0,23 & 0,40 & 0,40 & 0,30 \\
\hline $\mathrm{H}_{2} \mathrm{O}+$ & nd & nd & 11,00 & 11,00 & 13,50 \\
\hline $\mathrm{H}_{2} \mathrm{O}-$ & 8,65 & nd & 0,40 & 0,40 & nd \\
\hline $\begin{array}{l}\mathrm{P}_{2} \mathrm{O}_{5} \\
\text { Perda a }\end{array}$ & nd & 0,81 & nd & nd & nd \\
\hline $1000^{\circ} \mathrm{C}$ & 9,45 & nd & $n d$ & nd & nd \\
\hline \multicolumn{6}{|c|}{$\begin{array}{l}1 \text { - Pelotas fecais de chamosita da Baía de Aratu, BA } \\
2 \text { - Chamosita recente de Loch Etive, Escócia (Rohrlich et alli, 1969) } \\
3 \text { - Chamosita recente do delta do Niger, com impurezas de quartzo e } \\
\text { goetita (Porrenga, 1965) } \\
4 \text { - Chamosita do Mioceno da Nigéria, com impurezas de quartzo e } \\
\text { - goefita (Porrenga, 1965) } \\
\text { - Chamosita do Devoniano da Algéria, com impurezas de siderita } \\
\text { nd - Não-determinado } \\
\text { (Porrenga, } 1965 \text { ) }\end{array}$} \\
\hline
\end{tabular}

nética pós-deposicional, com condições locais redutoras (dentro da própria pelota fecal ou carapaça do foraminífero, por exemplo).

A maioria das conchas preenchidas apresenta-se total ou parcialmente dissolvida, indicando que o sedimento, num tempo mais ou menos recente, foi submetido à condições de $\mathrm{pH}$ ácido.

Na Baía de Aratu, a chamosita apresenta-se associada, nas pelotas e nos moldes dos organismos, à montmorilonita e ao quartzo. Ao lado disso, foram encontrados, na Baía de Aratu, outros minerais de ferro, como a goethita e a siderita.

Agradecimentos Os autores agradecem o apoio e incentivo dispensado pela professora Yeda de A. Ferreira, Coordenadora do Programa de Pesquisa e Pós-Graduação em Geofísica da UFBa, no âmbito do qual este trabalho foi realizado. Agradecimentos são extensivos ao Prof. Shiguemi Fujimori, pelas críticas e sugestões apresentadas, aos profs. Zelinda M. A. Nery Leão, Arno Brichta e Enrico Di Napoli (já falecido) e ao prof. Tersandro do Rego Monteiro, responsável pelo Laboratório de Raios X do Instituto de Geociências da UFBa. Por fim, são consignados agradecimentos às entidades financiadoras, FINEP e BNDE, que tornaram possivel a realização do trabalho.

\section{BIBLIOGRAFIA}

BRINDLEY, G. W. - 1961 - Kaolin, Serpentine and Kindred minerals, pp. 51-131, in Brown, G. ed., The X-Ray Identification and Crustal Structures of Clay Minerals. Mineral. Soc. London, $544 \mathrm{pp}$. 
BURST, J. F. - 1958 - Glauconite pellets: their mineral nature and applications to stratigraphic interpretations. Bull, Am. Ass. Petrol. Geol. 42(2): 310-327

MARTIN, L. - 1972 - Étude des "Faecal-Pellets" minéralisés des Sédiments du Plateau Continental de Côte D'Ivoire. Cah. ORSTOM, sér. Geol., IV(2): 105-120

PORRENGA, D. H. - 1965 - Chamosite in recent sediments of the Niger and Orinoco deltas. Geol. en Mijnb., 44: 400-403

PORRENGA, D. H. - 1967 - Glauconite and chamosite as depth indicators in the marine environment. Mar. Geol., 5(5/6): 495-501

ROHRLICH, V., PRICE, N. B. and GALVERT, S. E. - 1969 - Chamosite in the recent sediments of Loch Etive. Scotland, Jour. Sed. Pet., 39(2): 624-631

STRAKHOV, N. M. - 1957 - Méthodes d'étude des roches sédimentaires. Ann. Service Inform. Geol. Bureau Rech. Geol. Geoph. Miniéres, Tome I: 72.73

TRIPLEHORN, D. M. - 1966 - Glauconite provides good oil search data. World Oil, 162: 94-96 